\title{
Trauma pediátrico: Resultados de um estudo prospectivo em um hospital público
}

\section{terciário}

\author{
Pediatric trauma: Results of a prospective study in a public tertiary hospital \\ Traumatismo pediátrico: resultados de un estudio prospectivo en un hospital terciario público
}

Recebido: 02/05/2021 | Revisado: 11/05/2021 | Aceito: 13/05/2021 | Publicado: 29/05/2021

\author{
Alessandro Santos Ferreira \\ ORCID: https://orcid.org/0000-0003-1781-4759 \\ Universidade Tiradentes, Brasil \\ E-mail: alessandro180598@gmail.com \\ Camila Conceição Santos Temóteo \\ ORCID: https://orcid.org/0000-0002-6469-2996 \\ Universidade Tiradentes, Brasil \\ E-mail: camilacstemoteo@gmail.com \\ Adriana Barbosa de Lima Fonseca \\ ORCID: https://orcid.org/0000-0002-5774-2457 \\ Universidade Tiradentes, Brasil \\ E-mail: dra_adrilima@yahoo.com.br
}

\begin{abstract}
Resumo
Objetivos: determinar o perfil clínico-epidemiológico do trauma mecânico pediátrico em um hospital público terciário do município de Aracaju-SE; descrever as características do trauma como o mecanismo, o tipo e gravidade; e analisar o desfecho do trauma. Metodologia: trata-se de um estudo observacional, prospectivo, de base hospitalar pautado na análise documental de dados obtidos a partir dos prontuários com registros de crianças internadas vítimas de trauma mecânico. Resultados: com base nos dados obtidos, de um total de 62 participantes da pesquisa, o sexo masculino foi o mais acometido, com $(62,9 \%)$ do total, sendo a faixa etária mais acometida foi de 5 a 9 anos, com (40,3\% dos casos); 64,5\% dos pacientes chegaram por meios próprios ao hospital, tendo como principal mecanismo de trauma a queda, 75,8\% dos casos. O politraumatismo esteve presente em 19,4\% dos pacientes. Conclusão: a maior parte dos casos de trauma evoluiu para alta hospitalar e não houve necessidade de admissão em UTI. O trauma não é apenas de uma doença grave, mas um relevante problema de Saúde Pública, pelo fato de ser a principal causa de mortalidade pediátrica nos países desenvolvidos, além de determinar a presença de sequelas transitórias ou permanente que, consequentemente, podem trazer prejuízos ao desenvolvimento físico, social e psicológico do paciente.
\end{abstract}

Palavras-chave: Epidemiologia; Pediatria; Trauma Mecânico.

\begin{abstract}
Objectives: to determine the clinical-epidemiological profile of pediatric mechanical trauma in a public tertiary hospital in the municipality of Aracaju-SE; describe the characteristics of the trauma such as the mechanism, type and severity; and analyze the outcome of the trauma. Methodology: this is an observational, prospective, hospital-based study based on documentary analysis of data obtained from medical records with records of hospitalized children victims of mechanical trauma. Results: based on the data obtained, of a total of 62 research participants, the male gender was the most affected, with $(62.9 \%)$ of the total, with the most affected age group being 5 to 9 years old, with (40.3\% of cases); $64.5 \%$ of the patients arrived at the hospital by their own means, with falls as the main trauma mechanism, $75.8 \%$ of the cases. Polytrauma was present in $19.4 \%$ of patients. Conclusion: most trauma cases evolved to hospital discharge and there was no need for admission to the ICU. Trauma is not only a serious disease, but a relevant Public Health problem, as it is the main cause of pediatric mortality in developed countries, in addition to determining the presence of transient or permanent sequelae, which, consequently, can cause damage to the physical, social and psychological development of the patient.
\end{abstract}

Keywords: Epidemiology; Pediatrics; Mechanical Trauma.

\section{Resumen}

Objetivos: determinar el perfil clínico-epidemiológico del traumatismo mecánico pediátrico en un hospital público terciario del municipio de Aracaju-SE; describir las características del trauma como el mecanismo, tipo y gravedad; y analizar el resultado del trauma. Metodología: se trata de un estudio observacional, prospectivo, hospitalario basado en el análisis documental de datos obtenidos de historias clínicas con registros de niños hospitalizados víctimas de traumatismos mecánicos. Resultados: de acuerdo con los datos obtenidos, de un total de 62 participantes de la investigación, el género masculino fue el más afectado, con (62,9\%) del total, siendo el grupo de edad más afectado el de 5 a 9 años, con (40,3\%) de los casos); El 64,5\% de los pacientes llegó al hospital por sus propios medios, siendo las 
caídas el principal mecanismo traumático, el 75,8\% de los casos. El politraumatismo estuvo presente en el 19,4\% de los pacientes. Conclusión: la mayoría de los casos de trauma evolucionaron hasta el alta hospitalaria y no hubo necesidad de ingreso en UCI. El trauma no solo es una enfermedad grave, sino un problema de Salud Pública relevante, ya que es la principal causa de mortalidad pediátrica en los países desarrollados, además de determinar la presencia de secuelas transitorias o permanentes, que, en consecuencia, pueden ocasionar daños en el cuerpo, desarrollo social y psicológico del paciente.

Palabras clave: Epidemiología; Pediatría; Trauma mecánico.

\section{Introdução}

O trauma, de acordo com o Comitê de Trauma do Colégio Americano de Cirurgiões, é caracterizado por alterações estruturais ou desequilíbrio fisiológico decorrente da exposição aguda a várias formas de energia (Santos, 2016). Na pediatria, a lesão por trauma é considerada a principal causa de morte no mundo e, pode trazer prejuízos ao desenvolvimento da criança (WHO, 2018; Rosa, et al., 2018). Além disso, os acidentes são a segunda principal causa de hospitalização em pessoas menores de 15 anos de idade (Franciozi, 2008)

Algumas características particulares à criança podem aumentar ou diminuir os riscos de lesões por trauma (Waksman e Blank, 2014). As crianças apresentam proporções, formas, tamanhos e volumes de regiões, órgãos e estruturas peculiares que se relacionam às necessidades adaptativas para a idade (Tavano, 2008). Além das características inerentes à criança, dos acidentes e das violências na infância, apresentam-se também diferenças no que diz respeito ao sexo, à faixa etária, ao local de ocorrência e a maneira como ocorreram (Silva, et al., 2010; Harada, et al., 2003).

As lesões por trauma na infância representam sofrimentos, culpas e perdas para a família, sendo considerada uma problemática de cunho social (Canabarro, 2004). Portanto, o trauma na faixa etária pediátrica pode resultar em danos irreversíveis, sejam sociais, físicos ou psicológicos, e podem marcar definitivamente a vida do indivíduo, além da família e da sociedade (WHO, 2018).

Os acidentes na infância e adolescência são considerados um problema de saúde pública (CEINFO, 2017). O Sistema Único de Saúde (SUS) gastou no ano de 2019, R\$ 89.288.190,48 de reais com internações por causas externas (Boone, et al., 2018). As causas externas, têm importância quanto a ser causa de morte precoce e diversas incapacidades entre crianças, adolescentes e os adultos jovens (Lima, et al., 2018).

Determinar as características e identificar os fatores envolvidos no trauma pediátrico é de fundamental importância para encorajar medidas de promoção saúde e redução da incidência de tais agravos. Por fim, o presente estudo avaliou o perfil clinico-epidemiológico dos pacientes que foram atendidos por trauma mecânico pediátrico em um hospital público terciário da cidade de Aracaju-SE durante os anos de 2019-2020.

\section{Metodologia}

Neste projeto foi utilizada uma proposta metodológica quantitativa para realizar uma pesquisa de campo. Trata-se de um estudo observacional, prospectivo, de base hospitalar pautado na análise documental de dados obtidos a partir dos prontuários com registros de crianças internadas vítimas de trauma mecânico (Soares, et al, 2018).

A coleta dos dados ocorreu no período de agosto de 2019 a janeiro de 2020 por meio do emprego de um formulário, previamente estabelecido, composto por questões acerca do trauma mecânico na faixa etária pediátrica. O formulário abordava as seguintes variáveis: sexo, idade, zona, cuidador no momento do trauma, local e horário da ocorrência, horário, como chegou ao hospital, mecanismo do trauma, admissão na UTI, evolução, tempo de permanência hospitalar, sequelas e capacidade funcional.

Foram consideradas elegíveis somente os prontuários de pacientes de zero a 12 anos de idade, independente do sexo, admitidas no Hospital de Urgências de Sergipe por trauma mecânico. Os critérios de exclusão foram: prontuário com 
informações insuficientes e/ou ilegíveis. Os dados coletados foram armazenados em formulário eletrônico e compilados em planilhas pelo Programa Microsoft Excel ${ }^{\circledR}$ Office XP. Para a interpretação estatística dos dados foram realizados análise descritiva por intermédio de frequências absolutas, relativas e cálculo das médias e desvio padrão.

A pesquisa foi submetida e aprovada pelo Comitê de Ética da Universidade Tiradentes, com parecer favorável de número 3.292.296 e CAAE: 10151519.1.0000.5371 e foram respeitados todos os artigos da Resolução 466/12.

Os autores declaram não haver conflitos de interesse.

\section{Resultados}

Com base nos dados obtidos, de um total de 62 participantes da pesquisa, o sexo masculino foi o mais acometido (62,9\% das crianças), sendo a faixa etária mais acometida foi de 5 a 9 anos, com (40,3\% dos casos). Em relação à procedência, $35,48 \%$ dos pacientes eram provenientes do município de Aracaju e 64,5\% dos pacientes eram de zona urbana.

A mãe foi a principal cuidadora em 59,7\% dos casos e a maior parte das ocorrências foram em via pública (, 48,4\%)\%, e no turno da tarde (39\%). De acordo com o registro dos prontuários, $64,5 \%$ dos pacientes chegaram por meios próprios ao hospital, tendo como principal mecanismo de trauma a queda, $75,8 \%$ dos casos. O politraumatismo esteve presente em $19,4 \%$ dos pacientes. Os principais mecanismos de trauma por sexo e faixa etária são apresentados na Tabela 1

Tabela 1. Crianças vítimas de trauma mecânico, segundo faixa etária, sexo e mecanismo de trauma. HUSE. Aracaju-SE. Brasil, 2019-2020.

\begin{tabular}{|c|c|c|c|c|c|c|c|}
\hline & \multicolumn{2}{|c|}{ o a 4 anos } & \multicolumn{2}{|c|}{5 a 9 anos } & \multicolumn{2}{|c|}{$\begin{array}{c}10 \text { a } 12 \\
\text { anos }\end{array}$} & \multirow[t]{2}{*}{ Total } \\
\hline & $\mathbf{F}$ & $\mathbf{M}$ & $\mathbf{F}$ & $\mathbf{M}$ & $\mathbf{F}$ & $\mathbf{M}$ & \\
\hline Queda & 6 & 8 & 7 & 12 & 6 & 8 & 47 \\
\hline Atropelamento como pedestre & 1 & 2 & 0 & 2 & 1 & 0 & 6 \\
\hline Acidente de motocicleta & 1 & 0 & 0 & 3 & 0 & 0 & 4 \\
\hline Acidente com animal & 0 & 1 & 0 & 0 & 0 & 1 & 2 \\
\hline Atropelamento como ciclista & 1 & 0 & 0 & 0 & 0 & 0 & 1 \\
\hline Acidente de carro & 0 & 0 & 0 & 1 & 0 & 0 & 1 \\
\hline \multirow[t]{2}{*}{ Por arma branca } & 0 & 0 & 0 & 0 & 0 & 1 & 1 \\
\hline & 9 & 11 & 7 & 18 & 7 & 10 & \\
\hline Total & 12 & & 25 & & 17 & & 62 \\
\hline
\end{tabular}

F: sexo feminino M: sexo masculino. Fonte: Autores (2019-2020).

O trauma esquelético esteve presente na maioria dos pacientes $(69,4 \%)$, acometendo principalmente os ossos do antebraço em 30,23\% das crianças. A segunda lesão mais encontrada na pesquisa foi o trauma cranioencefálico, sendo frequente em $32,3 \%$ dos pacientes. Em seguida, foram encontrados o trauma muscular $(11,50 \%)$ e o trauma de face $(8,10 \%)$. O 
trauma abdominal e torácico estiveram presente em 2 casos, 3,2\% dos pacientes. O Gráfico 1 mostra a frequência da ocorrência de trauma conforme sua etiologia.

Gráfico 1. Frequência da ocorrência de trauma segundo etiologia. HUSE. Aracaju-SE. Brasil, 2019-2020.

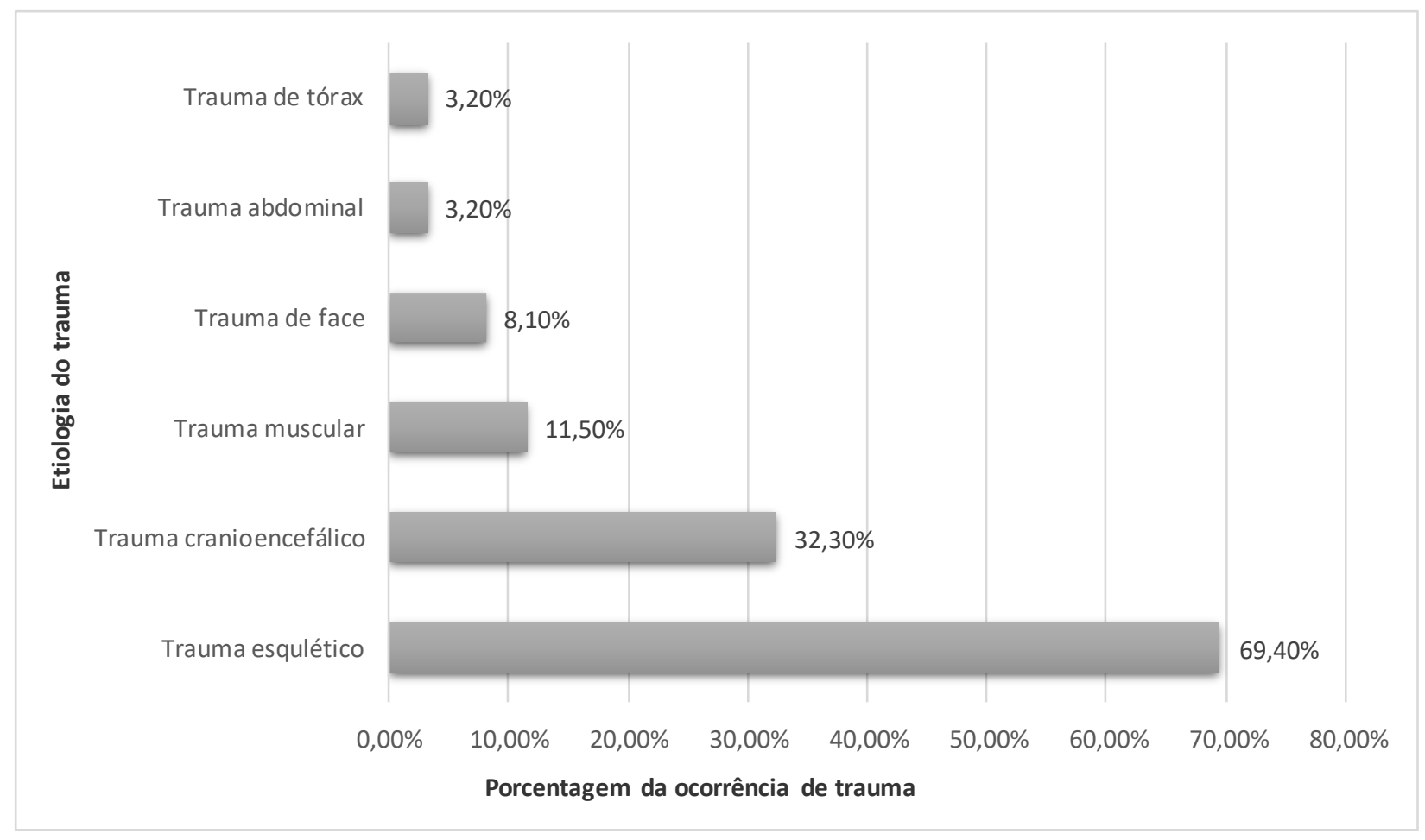

Fonte: Autores.

A frequência de admissão na UTI foi de 6,5\%. A média de permanência hospitalar foi de 5 dias e o percentual de alta hospitalar, por sua vez, foi de 98,4\% dos pacientes. Em relação à capacidade funcional, 67,7\% dos pacientes a mantiveram preservada enquanto $10,2 \%$ dos pacientes apresentaram sequelas, sendo a sequela motora o principal tipo (11,8\%). A taxa de óbito foi de $1,6 \%$ decorrente de ferimento por arma branca.

\section{Discussão}

No presente estudo foram analisados 62 prontuários de internações por trauma mecânico em crianças na faixa etária de 0 a 12 anos. Dessa forma, através dos resultados obtidos, foi possível observar maior frequência desse agravo em crianças do sexo masculino, achado que coaduna com os resultados descritos na literatura (Silva, et al., 2020; Luís et al., 2019).

Estudos realizados entre 2013 e 2015 em Brasília revelam que o resultado para idade e sexo é considerado relevante, visto que os dados encontrados evidenciaram maiores índices de ocorrências em pacientes do sexo masculino e, a faixa etária para ocorrência destes agravos foram em crianças a partir 3 a 12 anos (Silva, 2017).

Em relação ao local da ocorrência, a pesquisa demonstrou que, em geral, os traumas ocorreram em vias públicas e residências: locais onde acredita-se que as crianças permaneçam por mais tempo. Um estudo realizado por Silva (2017), encontrou resultados semelhantes, tendo como resultados $65,75 \%$ em vias públicas e $26,51 \%$ na residência. De acordo com a literatura, a maioria dos acidentes ocorre na residência pelo fato de apresentar grande variedade de objetos e situações de risco para a ocorrência de trauma (Vieira, 2013).

No que se refere à zona de ocorrência do trauma, a maior parte foi proveniente de região urbana. De acordo com 
Feliciano et al. (2008), a incidência e o tipo de lesão variam entre a população urbana e a rural, porém, óbitos por lesões não intencionais são comuns nas regiões rurais. Notam-se ainda variações também entre diferentes regiões de um país e conforme composição demográfica e meio físico, social e econômico.

De acordo com os prontuários analisados na pesquisa, o principal mecanismo de trauma foi a queda, o qual está em consonância com os resultados obtidos nos estudos de Silva et al. (2020) e Luís et al. (2019). Os fatores que influenciam a ocorrência de quedas no ambiente doméstico são multifatoriais: características como a idade ou gênero, o tipo de moradia, piso, espaço, tipo e tamanho dos móveis, tem impacto sobre a incidência e o padrão de lesões domésticas não intencionais (Oliveira, et al., 2018).

A principal lesão decorrente do trauma mecânico encontrada na pesquisa foi o trauma esquelético, acometendo, principalmente, os ossos do membro superior. Um estudo sobre trauma ortopédico realizado por Cantão et al. (2021) realizado em hospital do interior do Pará, obteve resultados compatíveis com o presente estudo. É perceptível que, na faixa etária escolar, a criança começa a provar a independência e interação com ao redor, logo, estão mais expostas à possíveis traumas ortopédicos (Pereira et al., 2013; Santili, et al., 2010)

Tais achados são de grande importância, tendo em vista que o trauma ortopédico, no público infantil, pode ocasionar sequelas irreversíveis, com consequência física, social ou psicológica (Cantão et al., 2021)

\section{Conclusão}

Segundo os resultados da pesquisa, a maior parte dos casos de trauma evoluiu para alta hospitalar e não houve necessidade de admissão em UTI. Aspectos importantes como dados sociodemográficos, causas mais incidentes de trauma na infância, mecanismos de trauma, principais complicações e desfecho do evento foram identificados no presente estudo.

Sabe-se que o trauma não é apenas uma doença grave, mas um relevante problema de Saúde Pública, pelo fato de ser a principal causa de mortalidade pediátrica nos países desenvolvidos, além de determinar a presença de sequelas transitórias ou permanente que, consequentemente, podem trazer prejuízos ao desenvolvimento físico, social e psicológico do paciente.

Por fim, é fundamental a realização de mais estudos para complementar o conhecimento acerca do trauma mecânico pediátrico, a fim de fundamentar ainda mais as ações de promoção da educação e de prevenção desses acontecimentos. Faz-se necessário a realização de pesquisas que abordem a temática do trauma pediátrico, para que esse grave problema de saúde pública seja melhor compreendido; e que políticas públicas de prevenção sejam adotadas.

\section{Referências}

Canabarro, S. T., Eidt, O. R., \& Aerts, D. R. G. D. C. (2004). Traumas infantis ocorridos em domicílio. Revista gaúcha de enfermagem= Revista gaucha de enfermería = Nursing journal of Rio Grande do Sul. 25(2), 257-265. http://hdl.handle.net/10183/23525.

Cantão, B. D. C. G., Júnior, A. A. V., dos Santos Cunha, E. L., Neto, J. B. D. S. B., de Almeida, C. L. S., dos Santos Silva, H. R., ... \& de Lima, A. B. (2021). Perfil Epidemiológico de traumas ortopédicos pediátricos em um hospital do interior do Pará. Revista Eletrônica Acervo Saúde, $13(2)$, e6265-e6265. https://doi.org/10.25248/reas.e6265.2021.

Centro de Epidemiologia e Informação (CEInfo). (2017). Epidemiologia e informação. https://www.prefeitura.sp.gov.br/cidade/secretarias/as ude/epidemiologia_e_informacao/index.php? $\mathrm{p}=19289$.

Boone, D. L., Costa, T. M. T., Tana, D. B., Lopes, S. C., Cortes, M. C. S., de Freitas, E. D., \& Alves, W. A. (2018). Serviço de Atendimento Móvel de Urgência: perfil dos atendimentos por causas externas. Revista da Sociedade Brasileira de Clínica Médica, 16(3), 134-139. http://www.sbcm.org.br/ojs3/index.php/rsbcm/article/view/352.

Feliciano, D. V., Matox, K. L., \& Moore, E. E. (2008). Trauma. (6a ed.), M Graw Hill, 25-38.

Franciozi, C. E. D. S., Tamaoki, M. J. S., De Araújo, E. F. A., Dobashi, E. T., Utumi, C. E., Pinto, J. A., \& Ishida, A. (2008). Trauma na infância e adolescência: Epidemiologia, tratamento e aspectos econômicos em um hospital público. Acta Ortopedica Brasileira, 16(5), 261-265. https://doi.org/10.1590/S1413-78522008000500001.

Harada, M. de J. C. S., Pedreira, M. da L. G., \& Andreotti, J. T. (2003). Segurança com brinquedos de parque infantil: uma introdução ao problema. Revista Latino-Americana de Enfermagem, 11(3), 383-386. https://doi.org/10.1590/s0104-11692003000300018. 
Lima, E. P. M. D., Almeida, A. O. A. D., Bezerra, E. P., Carneiro, E. P., Andrade, F. M. R. D., \& Gubert, F. D. A. (2018). Identificação dos conhecimentos de mães na prevenção de acidentes domésticos com crianças da primeira infância. Enferm. foco (Brasília), 77-80. http://biblioteca.cofen.gov.br/identificacaomaes-prevencao-acidentes-domesticos-criancas-primeira-infancia/.

Luiz, A., Braz, A., Fachini, J., Dias Júnio, G. r, \& Rodrigues, F. (2019). Home accidents in a pediatric emergency room in the South Region of Brazil. Residência Pediátrica, 9(2), 119-124. https://doi.org/10.25060/residpediatr-2019.v9n2-07.

Oliveira, M. J. S. de, Santos, F. Dos, Lange, C., Casagranda, L. P., Thumé, E., \& Castro, D. S. P. (2018). Acidentes por quedas e fratura do fêmur na população idosa. Revista de Enfermagem Da UFSM, 8(2), 225. https://doi.org/10.5902/2179769226168.

Pereira, I., Oliveira, C., Figueiredo, C., Cunha, D., Sales, G., \& Valença, A. (2013). Perfil das Internações de Crianças e Adolescentes com Fraturas do Crânio e Ossos da Face na Região Nordeste do Brasil. Revista Brasileira de Ciências Da Saúde, 17(3), 275-280. https://doi.org/10.4034/rbcs.2013.17.03.10.

Rosa, J. O., Azevedo, W. F., \& Florêncio, R. P. de S. (2018). Epidemiologia do trauma ortopédico pediátrico em um hospital público. Revista Científica Multidisciplinar Núcleo Do Conhecimento, 01(12), 166-173. https://doi.org/10.32749/nucleodoconhecimento.com.br/saude/trauma-ortopedico.

Santili, C., Gomes, C. M. de O., Akkari, M., Waisberg, G., Braga, S. dos R., Lino, W., \& Santos, F. G. (2010). Fraturas da diáfise da tíbia em crianças. Acta Ortopedica Brasileira, 18(1), 44-48. https://doi.org/10.1590/S1413-78522010000100009.

Santos, L. F. S., Fonseca, J. M. A., Cavalcante, B. L. S., \& Lima, C. M. (2016). Estudo epidemiológico do trauma ortopédico em um serviço público de emergência. Cad. Saúde Colet., 24 (4): 397-403. http://dx.doi.org/10.1590/1414-462x201600040128 .

Silva, C. V. F., Besborodco, R. M., \& Rodrigues, C. L., \& Górios C. (2020). Isolamento social devido à COVID-19 - epidemiologia dos acidentes na infância e adolescência. Resid Pediatr., 10(3):1-6 402. Disponível em: https://doi.org/10.25060/residpediatr.

Silva, M. A. I., Pan, R., Melo, L., de Bortoli, P. S., \& Nascimento, L. C. (2010). Perfil dos atendimentos a crianças e adolescentes vítimas de causas externas de morbimortalidade, 2000-2006. Revista Gaúcha de Enfermagem / EENFUFRGS, 31(2), 351-358. https://doi.org/10.1590/s1983-14472010000200021

Silva, V. B. (2017). Trauma pediátrico grave: análise da prevalência em hospital terciário do Distrito Federal, período de 2013 a 2015 . [Dissertação Mestrado em Enfermagem, Universidade de Brasília]. https://repositorio.unb.br/handle/10482/23917.

Soares, A., Dorlivete, P., Shitsuka, M., Parreira, F. J., \& Shitsuka, R. (2018). Metodologia da pesquisa científica. UFSM.

Tavano, T. P., (2008). Anatomia do recém-nascido e da criança: características gerais. Ensaios e Ciência: Ciências Biológicas, Agrárias e da Saúde, XII(1), 6375. https://www.redalyc.org/articulo.oa?id=26012806006.

Vieira, C. L. (2013). Ferimento transfixante em criança: relato de caso clínico. Rev. cir. traumatol. buco-maxilo-fac, 13(2), 57-61. http://revodonto.bvsalud.org/scielo.php?script=sci_arttext\&pid=S1808-52102013000200009.

Waksman, R. D., \& Blank, D. (2014). Prevenção de acidentes: um componente essencial da consulta pediátrica. Residência Pediátrica. 4(3) S36-S44. https://doi.org/10.25060/residpediatr.

World Health Organization (WHO). (2018). Global Reference List of 100 Core Health Indicators (plus health-related SDGs). https://www.who.int/healthinfo/indicators/2018/en/. 